\title{
A MODIFIED VAN DER POL EQUATION WITH DELAY IN A DESCRIPTION OF THE HEART ACTION
}

\author{
BEATA ZDUNIAK *, MAREK BODNAR **, URSZUla FORYŚ ** \\ ${ }^{*}$ Faculty of Applied Informatics and Mathematics \\ Warsaw University of Life Sciences, Nowoursynowska 159, 02-776 Warsaw, Poland \\ e-mail: beata.zduniak@wp.pl \\ ${ }^{* *}$ Faculty of Mathematics, Informatics and Mechanics \\ University of Warsaw, Banacha 2, 02-097 Warsaw, Poland
}

\begin{abstract}
In this paper, a modified van der Pol equation is considered as a description of the heart action. This model has a number of interesting properties allowing reconstruction of phenomena observed in physiological experiments as well as in Holter electrocardiographic recordings. Our aim is to study periodic solutions of the modified van der Pol equation and take into consideration the influence of feedback and delay which occur in the normal heart action mode as well as in pathological modes. Usage of certain values for feedback and delay parameters allows simulating the heart action when an accessory conducting pathway is present (Wolff-Parkinson-White syndrome).
\end{abstract}

Keywords: system with feedback, stability, action potential.

\section{Introduction}

The paper is related to the research on an electrical conduction system of the human heart. This system contains a sino-atrial node (SA), an atrio-ventricular node (AV) and the His-Purkinje system (Johnson, 1997). The two nodes can be modeled by a modified van der Pol oscillator (Żebrowski et al., 2008), while the His-Purkinje system can be treated as a passive delaying element. This model allows rendering phenomena observable in clinical experiments like Holter recordings.

Based on the van der Pol equation, we study the influence of feedback which occurs in the normal heart action mode as well as in pathological modes. Particularly, it is important to introduce time delay into this feedback. Properties of the modified model with delay are examined. Delay values used in our research correspond to those which occur in an electrical conduction system, for example, in the case of an accessory conducting pathway, the so-called Wolff-Parkinson-White syndrome. In the conduction system of the heart the only correct electrical conduction pathway between atria and ventricles is the bundle of His. Part of the population has abnormal accessory pathways through which electrical pulses are directly conducted from atria to ventricles. The atrio-ventricular node in a healthy heart ensures delay of electrical pulses generated in the sino-atrial node. The presence of an accessory conducting pathway may be the reason for AV nodal reentrant tachycardia occurrence. This pathway is called the bundle of Kent and conducts electrical pulses quicker than the atrio-ventricular node. In that case the wave of depolarization goes from atria to ventricles through the accessory path and returns to atria through the bundle of His. Such a wave of depolarization is called "reentry" and is the reason for tachycardia.

Atrio-ventricular nodal reentrant tachycardia (AVNRT) occurs when there are dual AV pathways conducting electrical impulses from atria to ventricles. The pathways join at two points within the AV node so as to form a circle. One pathway conducts impulses quickly but has a long refractory period. The other conducts impulses slowly but recovers quickly from depolarization. Because of the existence of the accessory conducting pathway outside the AV node, a flutter wave starts to circle. In our paper, the result of introducing feedback to the modified van der Pol system is creation of a wave similar to the initial wave-action potential, only delayed. It can be assumed that such feedback can be treated as an external, periodic excitation of the wave. 
The analysis of periodic orbits of various modifications of the van der Pol model has been the aim of many papers (Giacomin and Neukirch, 1997; Palit and Datta, 2010). Moreover, the model with delay and often with a term including the coupling coefficient has been considered in many articles (e.g., Erneux and Grasman, 2008; Jiang and Wei, 2008; Yu and Cao, 2005; Zhou et al., 2011). However, in these papers there are no examples of application of this model for recreating pathological behavior of the electrical-conduction system of the human heart, and therefore the ranges of parameters considered are wider than those that can be used in medical applications. Such wide ranges of the model parameters yield interesting qualitative results, e.g., Hopf bifurcation, Bogdanov-Takens bifurcation, transcritical and pitchfork bifurcations, but also solutions which are stable can be found. Erneux and Grasman (2008) showed that progressively stronger feedback for the weakly nonlinear oscillator leads to a large perturbation of the amplitude and possibly to a successive Hopf bifurcation. Bifurcation analysis was obtained by center manifold theory and the normal form method for functional differential equations (Jiang and Wei, 2008; Yu and Cao, 2005; Zhou et al., 2011). The van der Pol equation provides rich dynamical behavior which we would like to exploit in the modeling of the heart action.

\section{Construction of the model}

A prototype of nonlinear oscillators, which was originally devised as a model in electronic circuit theory, may be described by the well-known van der Pol equation (Kaplan and Glass, 1995),

$$
\ddot{x}+a\left(x^{2}-1\right) \dot{x}+x=0, \quad a>0,
$$

where $f(x)=\frac{1}{2} a\left(x^{2}-1\right)$ is a damping coefficient being a function of the $x$ variable, which is negative for $|x|<1$ and positive for $|x|>1$. Negative damping corresponds to the situation when energy is added to the system and the amplitude of oscillations grows, whereas positive one corresponds to the reduction of energy and the oscillation amplitude. This is a relaxation oscillator, which is very useful for description of the heart dynamics, because it easily adjusts its frequency to excitation frequency. The dynamics of Eqn. (1) are well known in the literature. This equation belongs to a wider class of Liénard's equations,

$$
\ddot{x}+F(x) \dot{x}+G(x)=0,
$$

where $G$ is odd and $F$ is even. Liénard's theorem gives sufficient conditions for the existence and uniqueness of a stable limit cycle (Palit and Datta, 2010).

Define $\Phi(x)=\int_{0}^{x} F(\xi) \mathrm{d} \xi$. Then these conditions are as follows:
1. $G(x)>0$ for $x>0$;

2. $\lim _{x \rightarrow \infty} \Phi(x)=\infty$;

3. there exists $\bar{x}>0$ such that $\Phi(x)<0$ for $0<x<\bar{x}$, $\Phi(x)>0$ for $x>\bar{x}$ and $\Phi$ is increasing.

It is obvious that Eqn. (1) is of the form (2). Moreover, it has only one steady state $x_{1}=0$, which is obviously unstable (a focus or a node depending on $a$ ), and all solutions are attracted by the limit cycle formed around this steady state. Two examples of the phase space portrait in the $(x, \dot{x})$ coordinates for $a=5$ and $a=0.5$ are presented in Fig. 2. The van der Pol model needs some changes in order to reproduce actual features of an action potential. Giacomin and Neukirch (1997) introduced modifications that maintain the required structure of the phase space. More precisely, the authors substituted a linear term by a nonlinear cubic force called the Duffing term,

$$
\ddot{x}+a\left(x^{2}-\mu\right) \dot{x}+\frac{x(x+d)(x+2 d)}{d^{2}}=0,
$$

where $a, \mu, d$ are positive control parameters.
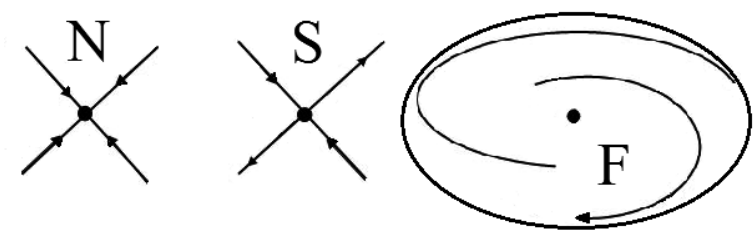

Fig. 1. Three steady states of the modified van der Pol oscillator: $\mathrm{N}$-stable node, $\mathrm{S}$-saddle, $\mathrm{F}$ - unstable focus surrounded by a limit cycle (Giacomin et al., 1997).

This model can be treated as an SA or AV node model. The mutual interaction of a limit cycle present around an unstable focus with a saddle, and a stable node is the main property of a modified relaxation oscillator, see Fig 1. As a result, the refraction period and nonlinear phase sensitivity of an action potential of node cells are reproduced correctly. A solution of this equation in time presents the action potential, whereas a solution in velocity allows obtaining a very important phase portrait. We can easily see that the main qualitative difference between Eqns. (1) and (3) is the appearance of two additional steady states, $x_{2}=-d$ and $x_{3}=-2 d$. As before, $x_{1}=0$ is an unstable node or a focus surrounded by a stable unique limit cycle, $x_{2}=-d$ is a saddle and $x_{3}=-2 d$ forms a focus or a node and can be either stable or unstable, depending on the sign of $4 d^{2}-$ $\mu$. In the case considered by Postnov (Giacomin et al., 1997) the first steady state is an unstable focus, while the third one is a stable node which attracts all solutions starting on the left hand-side of the stable manifold of 


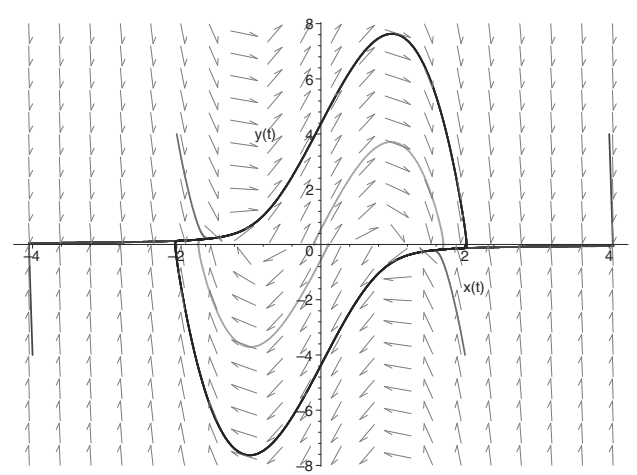

(a)

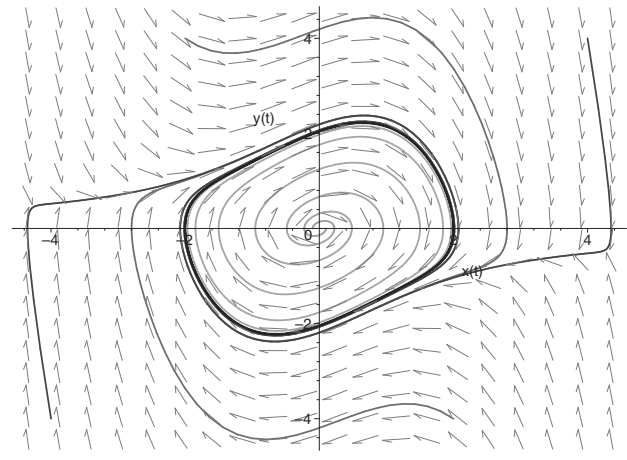

(b)

Fig. 2. Phase space portraits in $(x, y)=(x, \dot{x})$ coordinates for $a=5$ (a) and $a=0.5$ (b). We see that for small $a$ the velocity $\dot{x}$ does not change as much and as rapidly as for large $a$. However, $a=5$ is a biologically relevant value used in a description of the heart action in our analysis.

the saddle $x_{2}$. However, in the examined model (3) it is difficult to regulate the location of steady states in the phase space, so to reproduce the heart behavior a new parameter $e$ is introduced. Notice that this modification has no influence on the phase portrait, whereas we have the opportunity to modify the location of steady states for parameter values (5). In order to simplify frequency regulation and obtain the proper timescale, the ed factor in the denominator is substituted with an independent coefficient $f$ corresponding to a harmonic oscillator's frequency (Palit and Datta, 2010).

As we are mainly interested in phase space analysis, we present the final version of the model in its two variable first order form that reads

$$
\begin{aligned}
& \dot{x}=y, \\
& \dot{y}=-a\left(x^{2}-1\right) y-f x(x+d)(x+e) .
\end{aligned}
$$

The selection of appropriate parameters was done after a verification of the model by Grudziński (2007). The aim was to recreate physiological properties of the biological model using mathematical equations. The setup of particular parameters and acceptable variability ranges are briefly presented below. This information is essential for examining the stability of our setup because without such limitations the system could have completely different properties and would not recreate physiological properties.

A modification of the value of parameter $a$ increases time intervals between pulses but also changes their shapes, as shown in Fig. 2. Such behavior is inconsistent with physiological observations. This disadvantage of the vdP oscillator is related to the phase space structure. A modification of the $e$ parameter of the node location influences the distance between consecutive potential needles without changing their shapes. This means that the mutual position of a saddle and a node influences the time of spontaneous depolarization, which is one of the physiological mechanisms of regulation of the action potential generation frequency. The dependence of the oscillator considered on the parameter $e$ is nonlinear (Grudziński, 2007). Saturation of the curve is consistent with physiological observations because of the fact that the heart's ability to increase the rhythm frequency decreases for higher rhythm frequencies.

Parameter values for mvdP were chosen such that the oscillation's frequency corresponds to a real frequency of $\mathrm{SA}$ and $\mathrm{AV}$ nodes. The parameter $e$ plays a major role in the validation process of the model. The parameter $a$ is in the range $[0.5,6]$, the parameter $e$ belongs to the interval $[7,12]$ and the parameter $f$ may change only in the small range $[2.5,3]$. However, the reference model has the following parameter values:

$$
a=5, \quad f=3, \quad d=3, \quad e=7,
$$

which were used by Grudziński (2007).

Notice that the phase space portrait is qualitatively the same as for Eqn. (3). We present this portrait together with the sketch of the vector field in Fig. 3.

For the specific parameter values (5) we are able to calculate an invariant set containing the trivial steady state. This set has boundaries described by the following equations:

$$
\begin{aligned}
& y=2(x+3), \\
& y=6(x-2)+2, \\
& y=-4.8 x+20, \\
& y=-8.7(x-1.2)+19.04, \\
& y=-13.7(x-1.4)+17.3, \\
& y=-20.8(x-1.6)+14.56, \\
& y=-33.1(x-1.8)+10.4, \\
& y=-86.4(x-2)+3.78,
\end{aligned}
$$




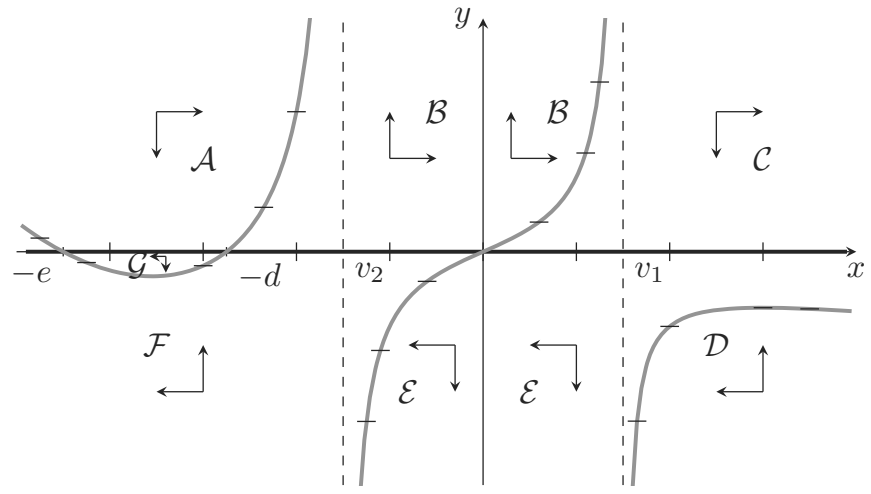

(a)

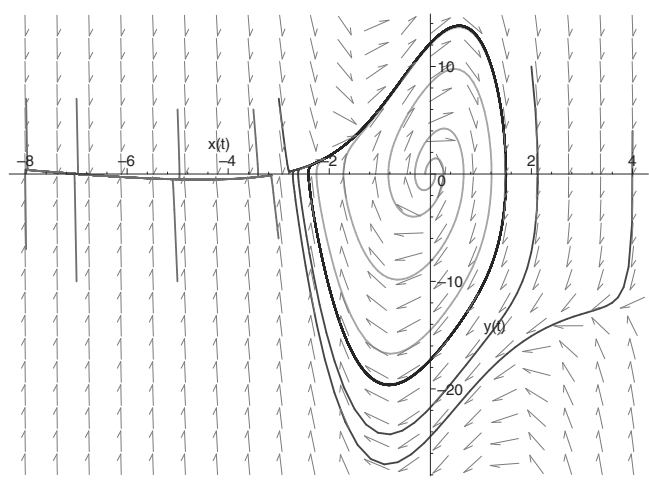

(b)

Fig. 3. Sketch of the vector field defined by Eqns. (4) (a) and the phase space portrait for the model parameters defined by (5) (b). The grey line is a null-cline for $y$ and the thick black line is a null-cline for $x$. Null-clines divide the phase plane into seven regions $\mathcal{A}, \mathcal{B}, \ldots, \mathcal{G}$. In each of these regions both functions $x(t)$ and $y(t)$ are monotonic.

$x=2.05$,

$y=5.7 x-25$,

$y=5 x$,

$y=2.2(x+0.6)-28$,

$x=-1$,

$y=-1.2(x+1)-29$,

$y=-4.7(x+1.3)-28.64$,

$y=-9.1(x+1.6)-27.23$,

$y=-14.3(x+1.9)-24.5$,

$y=-20.4(x+2.2)-20.21$,

$y=-27.4(x+2.5)-14.09$,

$y=-32.6(x+1.3)-8.61$,

$y=0$,

$$
\begin{aligned}
& y \in[-13.315,0], \\
& x \in[0,2.05], \\
& x \in[-0.6,0], \\
& x \in[-1,-0.6], \\
& y \in[-29,-28.88], \\
& x \in[-1.3,-1], \\
& x \in[-1.6,-1.3], \\
& x \in[-1.9,-1.6], \\
& x \in[-2.2,-1.9], \\
& x \in[-2.5,-2.2], \\
& x \in[-2.7,-2.5], \\
& x \in[-2.96412,-2.7], \\
& x \in[-3,-2.96412] .
\end{aligned}
$$

This allows us to conclude that due to the instability of the trivial steady state there exists a limit cycle located inside these boundaries.

In this paper we focus on the investigation of properties of the modified van der Pol equation with feedback and delay. Setting the problem in this way is important in the study of multi-diseases, e.g., the WPW syndrome and AVNRT. Pathology in action and conduction of the node in these illnesses is described in Introduction. In the model we study the feedback describes the situation when the output from the system influences the input and modifies the system action. In electronic engineering, in which mostly linear four-poles are considered, we can distinguish positive (in which a signal is amplified) and negative feedback loops. In nonlinear dynamics, feedback does not have to behave like that. Nonlinearity has a very serious impact on the behavior of a system (e.g, Maccari, 2001; Xu and Chung, 2003; Reddy et al., 2000; Liu et al., 2013).

Moreover, small delays are observed in the
WPW syndrome and AVNRT. The time when a wave goes along uncontrolled in order to return to the node (the time of flutter waves passing) is reflected by the delay which is added to the feedback part. We are interested in the range of delays which can occur at the existing accessory conducting pathway. We demonstrate that the feedback added to system in this way allows modelling real behavior of the heart in these kinds of pathologies.

Therefore, including the time delay in the feedback, we obtain the final version of our model that reads

$$
\begin{aligned}
\dot{x}= & y(t)+k(x(t-T)-x(t)), \\
\dot{y}= & -a\left(x^{2}(t)-1\right) y(t) \\
& -f x(t)(x(t)+d)(x(t)+e),
\end{aligned}
$$

where $k$ is a coupling coefficient, $T$ is a delay and other parameters are defined by (5).

\section{Analysis of Equations (6)}

At the beginning we prove the existence and uniqueness of solutions.

Theorem 1. Let $\varphi_{x}, \varphi_{y}$ be arbitrary continuous functions defined on the interval $[-T, 0]$. Then there exists a unique solution of Eqns. (6) defined on $[0,+\infty$ ).

Proof. We use the step method (Hale and Lunel, 1993) and study Eqns. (6) as a system of non-autonomous ordinary differential equations (ODEs) on the intervals of the delay length. Let us consider Eqns. (6) with an initial time-point $t_{0}=0$ and a continuous initial function $\varphi_{x}$ defined on $[-T, 0]$. Hence, the system considered may be solved on the interval $[0, T]$ as ODEs. Then, if the solution on $[0, T]$ exists, we can calculate it recursively for other intervals $[n T,(n+1) T]$. More precisely, on $[0, T]$ the 
system reads

$$
\begin{aligned}
\dot{x}= & y(t)+k\left(\varphi_{x}(t-T)-x(t)\right), \\
\dot{y}= & -a\left(x^{2}(t)-1\right) y(t) \\
& -f x(t)(x(t)+d)(x(t)+e) .
\end{aligned}
$$

We easily see that local solutions exist and are unique, as the autonomous part of the right-hand side is polynomial while the non-autonomous term $k \varphi_{x}(t-T)$ is continuous. We shall show that solutions exist on the whole interval $[0, T]$. Therefore, we need to exclude a blow up, that is, the existence of $\bar{t} \in[0, T]$ such that the solution tends to $\infty$ when $t \rightarrow \bar{t}^{-}$. Assume that such $\bar{t}$ exists. If $x$ is bounded, then $y \rightarrow \infty$, but this implies $\dot{x} \rightarrow \infty$, which contradicts the boundedness of $x$. If we set up that $y$ is bounded, then

$$
x(t)=x_{0}(0) \mathrm{e}^{-k t}+\int_{0}^{t}\left(y(s)+k \varphi_{x}(t-T)\right) \mathrm{e}^{k(s-t)} \mathrm{d} s
$$

is bounded as well. Hence, it suffices to show it is impossible that $\lim _{t \rightarrow \bar{t}} x(t)=\lim _{t \rightarrow \bar{t}} y(t)=\infty$. However, looking at the right-hand side of Eqns. (7), it is easy to see that

- if $x>0$ and $y>0$ sufficiently large, then $\dot{y}<0$, and therefore $y$ cannot tend to $+\infty$;

- if $x<0$ and $y<0$ with sufficiently large absolute values, then $\dot{y}>0$, and therefore $y$ cannot tend to $-\infty$;

- if $x<0$ and $y>0$ with sufficiently large absolute values, then $\dot{x}>0$, and therefore $x$ cannot tend to $-\infty$;

- if $x>0$ and $y<0$ with sufficiently large absolute values, then $\dot{x}<0$, and therefore $x$ cannot tend to $+\infty$.

Hence, unique solutions exist on the whole interval $[0, T]$ and can be prolonged to $\infty$ using the step method.

Now, we turn to the analysis of steady states. Examination of stability is essential for this kind of systems. For the specific parameters (5) it is possible to check stability using the Mikhailov criterion (Foryś, 2004).

Theorem 2. (Mikhailov criterion) Let a function $W: \mathbb{C} \rightarrow \mathbb{C}$ be of the form

$$
W(z)=\sum_{k=0}^{m} A_{k}(z) \mathrm{e}^{-h_{k} z},
$$

where $0=h_{0}<h_{1}<\cdots<h_{m}, A_{k}(z)=\sum_{j=0}^{n_{k}} a_{j k} z^{j}$, $a_{j k} \in \mathbb{R}, n_{0} \geq 1$ and $n_{k}<n_{0}$, for $k=1, \ldots, m$. If
$W(z)$ has no zeros on the imaginary axis, then all roots of $W$ lie in the open left half-plane of the complex plane if and only if $\Delta=n_{0} \pi / 2$, where $\Delta$ denotes the change in the argument of $W(i \omega)$ when $\omega$ increases from 0 to $\infty$.

Now, we use this theorem to check the stability of the steady states of Eqns. (6). Let $A_{i}=f\left(x_{i}\left(x_{i}+e\right)+\right.$ $\left.x_{i}\left(x_{i}+d\right)+\left(x_{i}+d\right)\left(x_{i}+e\right)\right), B_{i}=a_{i}\left(x_{i}^{2}-1\right) i=1,2,3$, and $x_{1}=0, x_{2}=-d, x_{3}=-e$. The characteristic quasi-polynomial for Eqns. (6) for the steady state $\left(x_{i}, 0\right)$ has the following form:

$$
\begin{aligned}
W(\lambda)= & \lambda^{2}+\left(B_{i}+k\right) \lambda+A_{i}+k B_{i} \\
& -k\left(\lambda+B_{i}\right) \mathrm{e}^{-\lambda T} .
\end{aligned}
$$

The curve drawn by the vector $W(i \omega)=$ $(\operatorname{Re}(W(i \omega)), \operatorname{Im}(W(i \omega)))$ in the complex plane as $\omega$ increases from 0 to $\infty$, is called the Mikhailov hodograph. The change in the shape of Mikhailov hodographs with increasing $T$ for $x_{1}$ is shown in Fig. 4 while for $x_{3}$ in Fig. 5

For both the steady states the shape of hodographs becomes more and more looped, but the total change in the argument of $W(i \omega)$ remains the same. However, for $x_{1}$ it is equal to $-\pi$, implying instability, while for $x_{3}$ it is $+\pi$, implying stability independently of the magnitude of the delay. As the steady state $\left(x_{2}, 0\right)$ is a saddle, it does not change stability with increasing delay.

On the other hand, we are also interested in the possible change in stability for other parameter values, and below we formulate a stricter result.

\section{Theorem 3.}

(i) The steady state $(0,0)$ of Eqns. (6) is an unstable focus for $T=0$. If

$$
\frac{\left|B_{1}\right|}{8 A_{1}}\left(4 A_{1}-B_{1}^{2}\right)<k<\frac{A_{1}}{2\left|B_{1}\right|},
$$

then stability switches can occur, that is, there may exist $k \geq 0$ and a sequence of numbers $0<T_{1}<T_{2}<$ $\cdots<T_{2 k-1}<T_{2 k}$ such that the steady state $(0,0)$ is locally asymptotically stable for $T \in\left(T_{2 j-1}, T_{2 j}\right), j=$ $1,2, \ldots, k$, and is unstable for $T \in\left(0, T_{1}\right) \cup\left(T_{3}, T_{4}\right) \cup$ $\cdots \cup\left(T_{2 k},+\infty\right)$. If

$$
k<\frac{\left|B_{1}\right|}{8 A_{1}}\left(4 A_{1}-B_{1}^{2}\right)
$$

or

$$
k>\frac{A_{1}}{2\left|B_{1}\right|},
$$

then the steady state $(0,0)$ is unstable independently of $T \geq 0$.

(ii) The steady state $(-d, 0)$ of Eqns. (6) is unstable independently of $T \geq 0$.

(iii) The steady state $(-e, 0)$ of Eqns. (6) is stable independently of $T \geq 0$. 


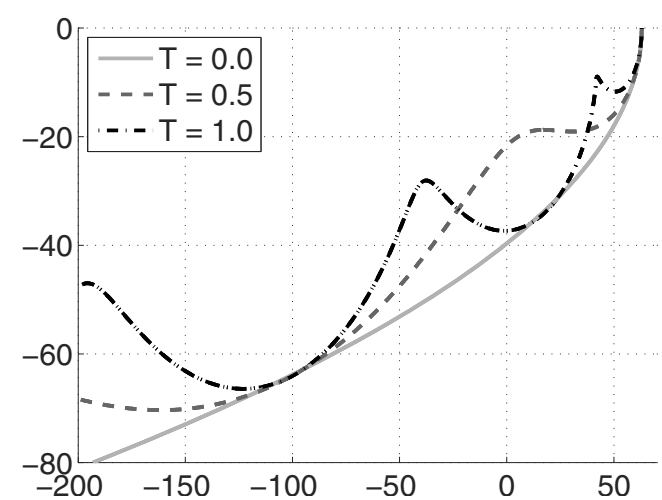

(a)

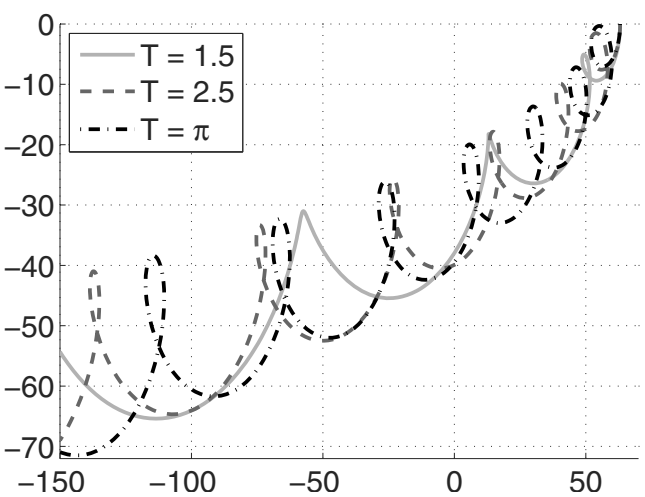

(b)

Fig. 4. With increasing delay $T$ the shape of Mikhailov hodograps for $x_{1}$ becomes more and more looped. However, the total change in the argument remains the same and equals $-\pi$, independently of the value of the delay, that is, $T=0,0.5,1$ (a) and $T=1.5$, $2.5, \pi(b)$.

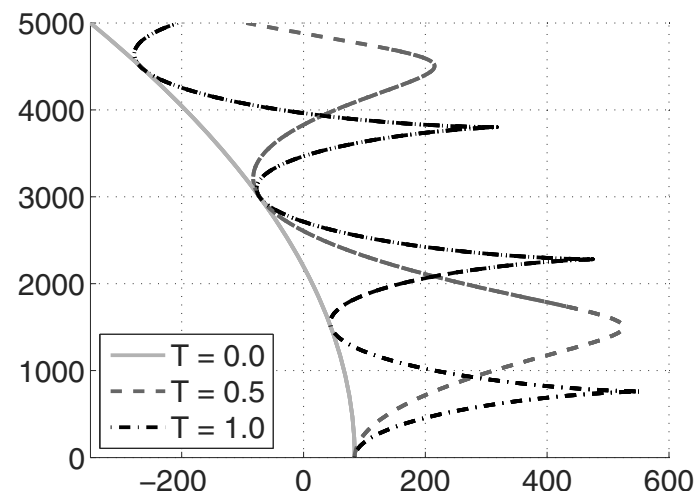

(a)

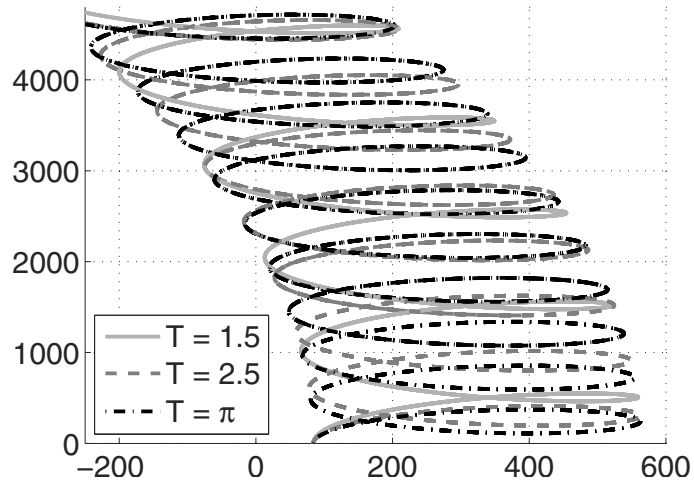

(b)

Fig. 5. With increasing delay $T$ the shape of Mikhailov hodograps for $x_{3}$ becomes more and more looped. However, the total change in the argument remains the same and equals $+\pi$, independently of the value of the delay, that is, $T=0,0.5,1$ (a) and $T=1.5$, $2.5, \pi(b)$.

Proof. The Mikhailov criterion implies that, if a steady state is a saddle point for $T=0$, then it does not change stability with increasing delay (Bielczyk et al., 2012). Therefore, the part (ii) is proved.

Now, we study the steady states $(0,0)$ and $(-e, 0)$, that is, an unstable focus and a stable node for $T=0$. We use the method presented by Cooke and van den Driessche (1986). If we rewrite the characteristic quasi-polynomial (8) in the form

$$
W(\lambda)=P(\lambda)+Q(\lambda) \mathrm{e}^{-\lambda T},
$$

and define the auxiliary function

$$
\begin{aligned}
F\left(\omega^{2}\right) & =\|P(i \omega)\|^{2}-\|Q(i \omega)\|^{2} \\
& =\omega^{4}+\left(B_{i}^{2}-2 A_{i}\right) \omega^{2}+A_{i}^{2}+2 k A_{i} B_{i},
\end{aligned}
$$

then, if $F$ has no positive zeros, any change in stability is impossible. On the other hand, if $F$ has at least one positive zero and each one is single, then stability changes may occur. Moreover, there exists $T^{*}$, such that the characteristic quasi-polynomial is unstable for all $T>T^{*}$ and when $T$ changes from 0 to $T^{*}$ the number of stability changes is finite.

The change of stability may occur if

I. $A_{i}^{2}+2 k A_{i} B_{i}<0$,

II. $A_{i}^{2}+2 k A_{i} B_{i}>0$ but $B_{i}^{2}-2 A_{i}<0$ and $\Delta=$ $\left(B_{i}^{2}-2 A_{i}\right)^{2}-4\left(A_{i}^{2}+2 k A_{i} B_{i}\right)=B_{i}^{4}-4 A_{i} B_{i}^{2}-$ $8 k A_{i} B_{i}>0$.

For the third steady state with $x_{3}=-e$ we have $A_{3}=$ $f e(e-d)>0$ and $B_{3}=a\left(e^{2}-1\right)>a\left(d^{2}-1\right)>0$. It is a stable node for $T=0$. Hence, $B_{3}^{2}-4 A_{3}>0$ in this case, and therefore neither the condition I nor the condition II of stability switches can be satisfied for this state.

For the first steady state with $x_{1}=0$ we have $A_{1}=f d e>0$ and $B_{1}=-a<0$. Notice that 
for $T=0$ its characteristic equation takes the form $\lambda^{2}+B_{1} \lambda+A_{1}=0$ and since we require this steady state to be an unstable focus, there must be $B_{1}^{2}<4 A_{1}$. Notice that, if $A_{1}^{2}+2 k A_{1} B_{1}<0$, then $F$ has a unique positive root $\omega_{0}^{2}>0$ and $F^{\prime}\left(\omega_{0}^{2}\right)>0$. Thus, eigenvalues cross the imaginary axis from left to right (Cooke and van den Driessche, 1986) and stability switches are impossible. Therefore, only the condition II can be taken into account, and in this case $B_{1}^{2}<2 A_{1}<4 A_{1}$, yielding that the condition for focus is satisfied.

Thus, the necessary conditions to stabilize the steady state $x_{1}=0$ are

(C1) $A_{1}^{2}+2 k A_{1} B_{1}>0$

(C2) $B_{1}^{2}-2 A_{1}<0$;

(C3) $\Delta=\left(B_{1}^{2}-2 A_{1}\right)^{2}-4\left(A_{1}^{2}+2 k A_{1} B_{1}\right)=B_{1}^{4}-$ $4 A_{1} B_{1}^{2}-8 k A_{1} B_{1}>0$.

Notice that for $k=0$ we have $\Delta=\left(B_{1}^{2}-4 A_{1}\right) B_{1}^{2}<$ 0 due to the condition (C2). Moreover,

$$
\Delta>0 \Longleftrightarrow \tilde{\Delta}(k)=B_{1}^{2}-4 A_{1}-8 \frac{A_{1}}{B_{1}} k>0 .
$$

In our case $B_{1}<0$ and this implies that the function $\tilde{\Delta}(k)$ is increasing. The condition $(\mathrm{C} 1)$ is satisfied as long as $k<-A_{1} /\left(2 B_{1}\right)$. Hence, the condition of stability switches for $x_{1}$ is given by the inequalities (9). It can be easily seen that for any $A_{1}>0$ and $B_{1}<0$ the inequality

$$
\frac{\left|B_{1}\right|}{8 A_{1}}\left(4 A_{1}-B_{1}^{2}\right) \leq \frac{A_{1}}{2\left|B_{1}\right|}
$$

is satisfied, and therefore the condition (9) defines a non-empty set.

Theorem 3 implies that for some parameter values stability switches may occur. The critical value of $T$ for which a stability switching is possible can be calculated as follows. Let $\omega_{j}>0$ be a solution of (11). Then, following Cooke and van den Driessche (1986), critical values of $T$ fulfil

$$
\begin{aligned}
& \cos \left(T \omega_{j}\right) \\
& =-\frac{\operatorname{Re}\left(P\left(i \omega_{j}\right)\right) \operatorname{Re}\left(Q\left(i \omega_{j}\right)\right)+\operatorname{Im}(P(i \omega)) \operatorname{Im}\left(Q\left(i \omega_{j}\right)\right)}{\operatorname{Re}\left(Q\left(i \omega_{j}\right)\right)^{2}+\operatorname{Im}\left(Q\left(i \omega_{j}\right)\right)^{2}} \\
& \sin \left(T \omega_{j}\right) \\
& =\frac{\operatorname{Re}\left(Q\left(i \omega_{j}\right)\right) \operatorname{Im}\left(P\left(i \omega_{j}\right)\right)-\operatorname{Re}\left(P\left(i \omega_{j}\right)\right) \operatorname{Im}\left(Q\left(i \omega_{j}\right)\right)}{\operatorname{Re}\left(Q\left(i \omega_{j}\right)\right)^{2}+\operatorname{Im}\left(Q\left(i \omega_{j}\right)\right)^{2}}
\end{aligned}
$$

where

$$
\begin{aligned}
& P\left(i \omega_{j}\right)=A_{i}+k B_{i}-\omega_{j}^{2}+i \cdot \omega_{j}(B+k), \\
& Q\left(i \omega_{j}\right)=k B+i \cdot k \omega_{j} .
\end{aligned}
$$

Thus, for the steady state $(0,0)$, we arrive at

$$
\begin{aligned}
& \cos \left(T \omega_{j}\right)=-\frac{k \omega_{j}^{2}+B_{1}\left(A_{1}+k B_{1}\right)}{k\left(\omega_{j}^{2}+B_{1}^{2}\right)}, \\
& \sin \left(T \omega_{j}\right)=\frac{\omega_{j}\left(\omega_{j}^{2}+B_{1}^{2}-A_{1}\right)}{k\left(\omega_{j}^{2}+B_{1}^{2}\right)},
\end{aligned}
$$

$j=1,2$, and we require that $T \omega_{j} \in[0,2 \pi)$

For parameters values given in (5) the inequalities (9) are fulfilled for $k \in\left(k_{\min }, k_{\max }\right)$, where $k_{\min }=$ $1135 / 504 \approx 2.25$ and $k_{\max }=6.3$. It turns out that for these values of parameters there exists a small interval of $T$ for which the trivial steady state can gain local stability.

In Fig. 6 we present the graphs of the critical value of $T$. The solid line denotes the critical value of $T$ for which eigenvalues cross the imaginary axis from right to left, while the dashed line denotes critical values for which eigenvalues cross the imaginary axis from left to right. In Fig. 8 we present sample solutions for the case when stability switches occur. However, if we change the value of $a$ or $f$, the interval $\left(k_{\min }, k_{\max }\right)$ changes. The upper end of the interval, $k_{\max }$, is a decreasing function of $a$ and an increasing function of $f$. The dependence of $k_{\min }$ on $a$ and $f$ is more complicated. It is an increasing function of $f$. The lower end of the interval, as a function of $a$, has a maximum for $\bar{a}=2 \sqrt{f d e / 3}$. In fact, for $a=0.5$ we have $k_{\min }=1007 / 4032 \approx 0.25$ and $k_{\max }=63$. For $a \approx 2.04$ (and the rest of parameters as in $(5)$ ) we have $k_{\text {min }}<1$, and several stability changes may occur for $k \approx 1$, which is presented in Fig. 7

Summarizing, in all cases we do not observe changes in the stability of a solution for the mvdP model for biologically relevant parameter values, as $k \approx 1$ is physiological. This means that the mvdP model with a given range of parameters values has very stable dynamics. It is very important in a dynamical system which describes a real medical problem, because the parameters of realistic models have observational errors. The model's stability also means that errors of the floating-point computations would not have a significant impact on the result.

\section{Numerical analysis}

For a numerical analysis of the discussed system, a numerical model was created using Dynamics Solver and a program in $\mathrm{C}++$ was written. A Dormand-Prince 8 integration algorithm was used. This method is a modification of the explicit Runge-Kutta formula with a variable integration step. Results were confirmed and plots were made with the Matlab dde23 solver. For the single node model of an electrical conduction system with no feedback, a periodic potential and the corresponding limit cycle (with a transient state) are obtained like in Fig. 9 The potential period equals 1.4 . 

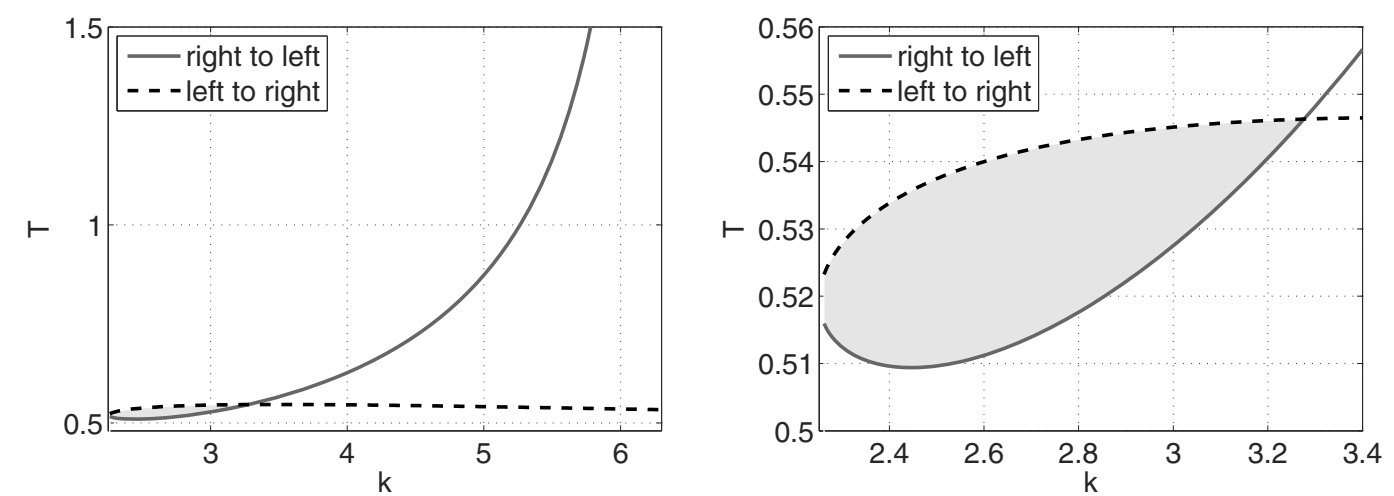

Fig. 6. Dependence of the critical value of the delay on $k$. The solid line denotes the critical value of $T$ for which eigenvalues cross imaginary axes from right to left, while the dashed line denotes critical values for which eigenvalues cross imaginary axes from left to right. The shaded area is a stability region.
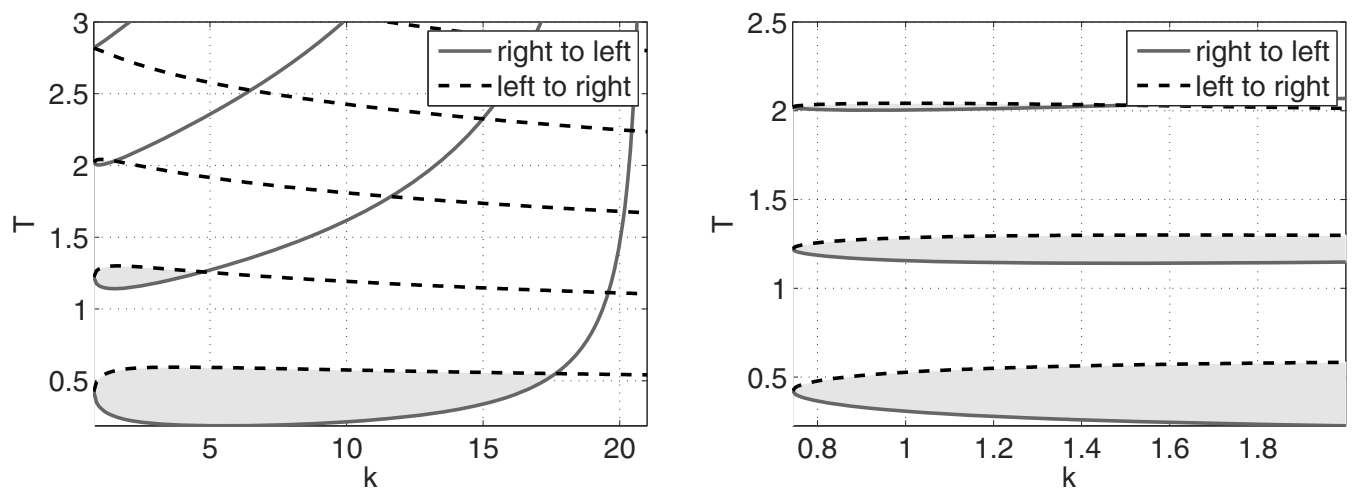

Fig. 7. Dependence of the critical value of the delay on $k$ for $a=1.5$ and the rest of parameters as in (5). The solid lines denote the critical value of $T$ for which eigenvalues cross imaginary axes from right to left, while the dashed lines denote critical values for which eigenvalues cross imaginary axes from left to right. The shaded area is a stability region.

After including feedback with $k=1$ and delay $T=$ 0.5 , time series look like on the given charts presented in Figs. 9 A solid line presents the modified model with the feedback and delay equal to 0.5 . For $T=1$, results are very similar. We can see that in the modified model the oscillation amplitude is reduced and the resting potential raised. This is caused by the delay, because the feedback itself does not influence the amplitude. Similar results were obtained (Atay, 1998) for a simple van der Pol oscillator with feedback.

In the case above the oscillation period is shortened and the limit cycle is decreased-the trajectory in one period is shorter, as shown in Fig. 9 We can see that the trajectory around the saddle moves slightly further from it and has a smoother shape. This means that the attraction by the stable manifold of the saddle point has decreased. Also the oscillation period is shortened. For the basic model (without feedback) the period equals 1.4, whereas when feedback and delay are included the period equals 0.8 or 0.9 .

In order to verify the influence of the feedback we set a small delay. For $k=1$ and $T=0.001$, results are the same as for the modified van der Pol model. For $k=1$ and $T=0.01$ we can observe, from a certain moment, a change in the period and with displacement of the oscillations to right compared with the basic one. Small feedback and delay parameter values do not modify the system's behavior much, but allow us to find out that types of fixed points do not change and the trajectory changes insignificantly. This means that for such small delay the feedback trajectories in the $(x, y)$ space remain the same and look the same as for the modified van der Pol equation. For $k=0.5$ and $k=2$ and delays like in the previous case we can observe insignificant influence of feedback. For a bigger feedback value there is a transient change in the period length which slightly disturbs the oscillator's action, but these changes are not big enough to modify its properties in long time.

The result of introducing feedback to the system is creation of a wave similar to the initial wave-the action potential, only delayed. It can be assumed that such feedback can be treated as an external, periodic excitation 


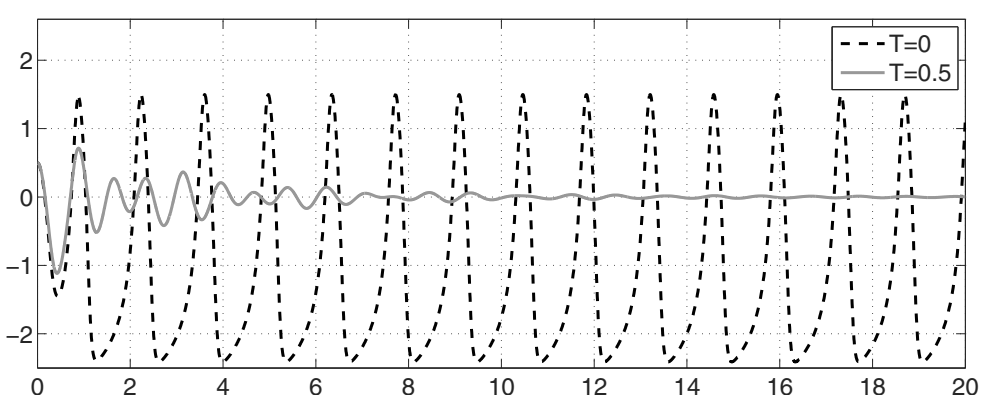

(a)

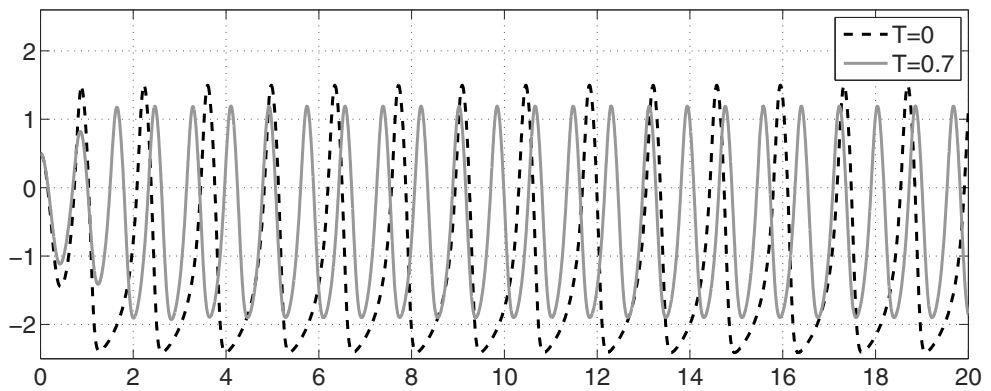

(c)

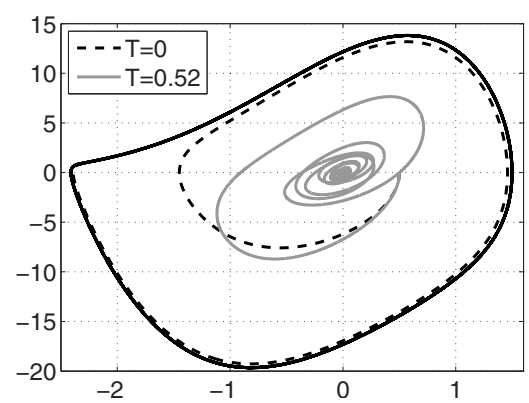

(b)

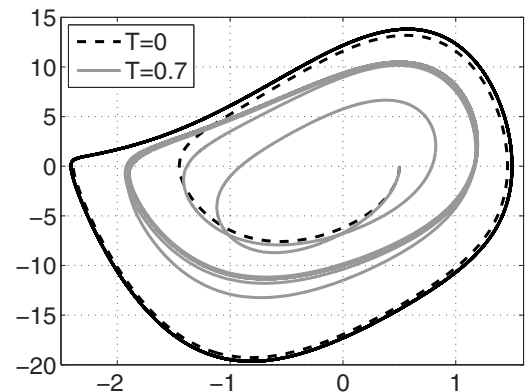

(d)

Fig. 8. Periodic potential for the single node model without feedback (dashed line with a bigger period) and for the model with feedback (solid line with a smaller amplitude) for $k=2.545$ and $T=0.5$ (upper line) and $T=0.7$ (lower line) (a), (c). Corresponding phase portraits (b), (d).

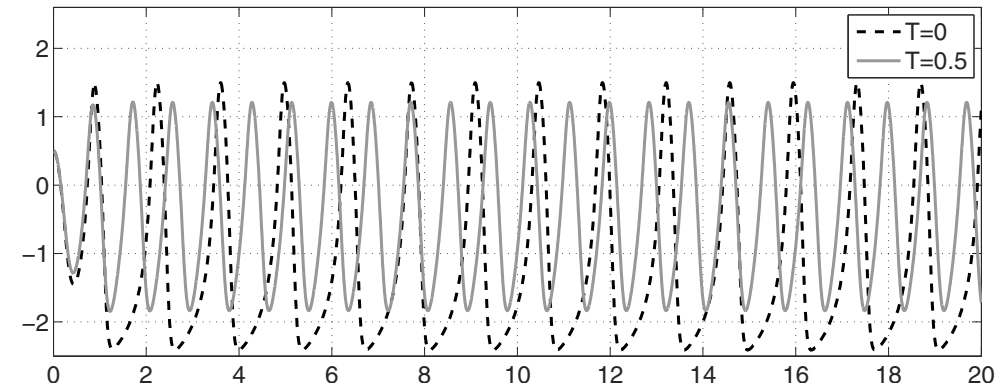

(a)

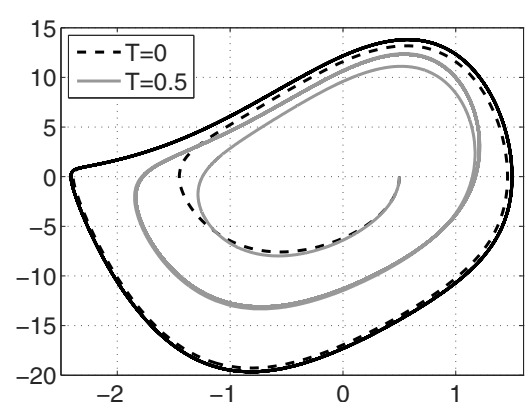

(b)

Fig. 9. Periodic potential for the single node model without feedback (dashed line with a bigger period) and for the model with feedback (solid line with a smaller amplitude) for $k=1$ and $T=0.5$ (line with a smaller amplitude) (a), phase portrait for the model without feedback (exterior line), with feedback $k=1$ and $T=0.5$ (interior line) (b).

of the wave. Period lengths of the model with feedback are constant. This model's behavior was analyzed by time series. We verified changes in the period length for relatively small delay values. The obtained results are regular and the period length of the oscillation does not change. The conclusion is that regular excitation may evoke a regular answer.

After examining the influence of excitation with the mvdP oscillator wave, we analyzed the behavior of the mvdP oscillator with feedback excited with a single rectangular pulse. In this way two excitations coexist in the system - the wave brought by the oscillator's feedback and the one from the external pulse.
The pulse is defined by a step function $H$ for the amplitude $A=4$ and the pulse length $L=0.05$, and is applied to the oscillator with $k=1$ and $T=1$. The function $H$ is defined as

$$
H_{A, L}(t)= \begin{cases}A & \text { if } 0<t<L \\ 0 & \text { otherwise }\end{cases}
$$

The influence of the pulse is treated as an appearance of the accessory current in our system. For this purpose, this pulse is introduced to the equation describing potential 
changing (the $x$ variable):

$$
\begin{aligned}
\dot{x}= & y(t)+k(x(t-T)-x(t))+H_{A, L}\left(t-t_{p}\right), \\
\dot{y}= & -a\left(x^{2}(t)-1\right) y(t) \\
& -f x(t)(x(t)+d)(x(t)+e),
\end{aligned}
$$

where $t_{p}$ denotes the time moment when the pulse starts.

The influence of the pulse, which results in a period length change, depends on a phase of oscillations in which it is applied. As mentioned before, the excitation influences the system having regular behavior.

A numerical solution of the system with feedback ( $k=1, T=0.5$ ), excited with the rectangular pulse, is presented in Fig. 10, The dashed line corresponds to the mvdP with feedback, whereas the solid one reflects the mvdP with feedback, which is additionally excited with the rectangular pulse.

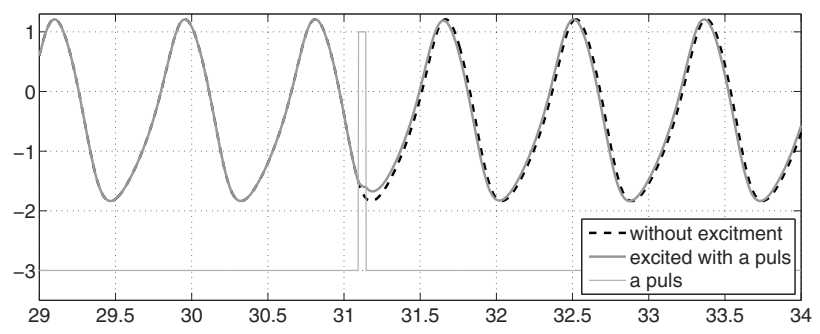

Fig. 10. Numerical solution of the system with feedback $(k=$ $1, T=0.5$ ), excited with the rectangular pulse where $A=4$ and $L=0.05$. The thin grey line indicates when the pulse given by $H_{A, L}$ is applied.

In this case, application of the external pulse does not influence the oscillator's period length and the oscillations are stable. There are phase ranges for which oscillations are sensitive to a disturbance. Phase response curves for the mvdP system without feedback and for two cases with feedback ( $k=1, T=1$ and $k=1, T=0.5)$ which are excited with the rectangular pulse ( $A=4, L=0.05$ ) are shown in Fig. 11. A phase response curve is a graphic presentation of the influence of excitation of oscillation phases of the mvdP system. Its shape depends on the phase of oscillation on which the excitation is put. This curve describes the dependence between the change in the length of one cycle of oscillation and the excitation phase $\phi$. For example, the application of excitation when the system abides in refraction state does not change its behavior.

The system is phase sensitive and that excitation, depending on the phase, may change the potential period length. Results of this analysis were confirmed experimentally. Models with feedback are much less phase sensitive, Fig. 11. Feedback strongly modifies properties of the mvdP model.

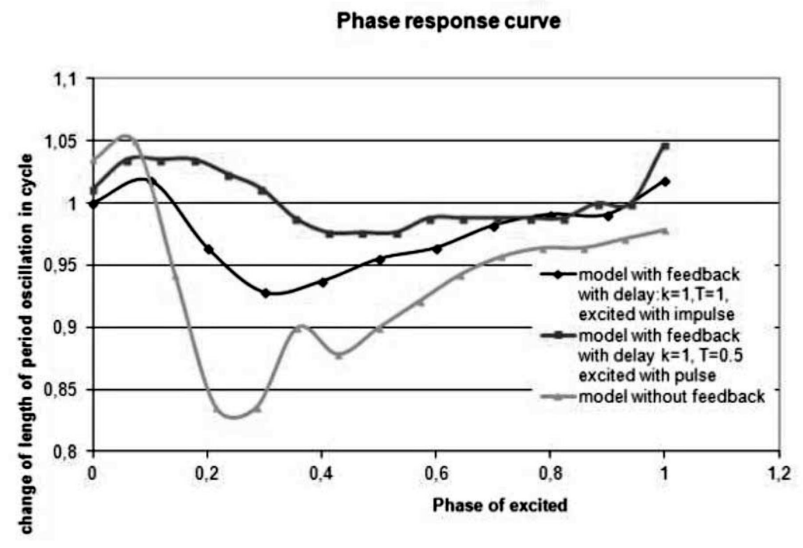

Fig. 11. Phase response curves for models with the rectangular pulse.

\section{Conclusions}

The human heart rhythm with feedback for the AV node is low-varying. The results of this study were consulted with a medical doctor from the Institute of Cardiology in Anin near Warsaw, who confirmed that a very stable heart rhythm is typical for patients with "AV nodal reentrant tachycardia". For many systems, feedback may stabilize oscillations. It is interesting that the nonlinear system starts to behave like a linear four-pole after applying feedback. Using only one feedback parameter $k$ may be questionable since the electrical pulse goes through different structures which have varying thicknesses. That is why the model with two different $k$ values was considered (the pulse going through two different tissues). The obtained result was insignificantly different from the one for one $k$ value. As a result of feedback and delay, the oscillation amplitude decreases and the resting potential (potential minimum) raises. This is caused by the delay only, and feedback does not affect that. Applying feedback and delay (large $k$ and $T$ ) results in changes in the phase portrait. Our model reconstructs correctly pathologies of the heart action like AVNRT or one of the WPW syndrome types. The system keeps local stability which is very important for mathematical models used for a description of medical phenomena.

\section{References}

Atay, F. (1998). Van der Pol's oscillator under delayed feedback, Journal of Sound and Vibration 218(2): 333-339.

Bielczyk, N., Bodnar, M. and Foryś, U. (2012). Delay can stabilize: Love affairs dynamics, Applied Mathematics and Computation 219(2): 3923-3937.

Cooke, K. and van den Driessche, P. (1986). On zeroes of some transcendental equations, Funkcialaj Ekvacioj 29(2): 77-90. 
Erneux, T. and Grasman, J. (2008). Limit cycle oscillators subject to a delayed feedback, Physical Review E 78(2): 026209-1-8.

Foryś, U. (2004). Biological delay systems and the Mikhailov criterion of stability, Journal of Biological Systems 12(1): 45-60.

Giacomin, H. and Neukirch, S. (1997). Number of limit cycles of the Lienard equation, Physical Review E 56(3809): 3809-3813.

Grudziński, K. (2007). Modeling the Electrical Activity of the Heart Conduction, Ph.D. thesis, Warsaw University of Technology, Warsaw.

Hale, J. and Lunel, S. (1993). Introduction to Functional Differential Equations, Springer-Verlag, New York, NY

Jiang, W. and Wei, J. (2008). Bifurcation analysis in van der Pol's oscillator with delayed feedback, Journal of Computational and Applied Mathematics 213(2): 604-615.

Johnson, L. (1997). Essential Medical Physiology, Lippincott Williams and Wilkins, London.

Kaplan, D. and Glass, L. (1995). Understanding Nonlinear Dynamics, Springer, New York, NY.

Liu, Y., Yang, R., Lu. J. and Cai, X. (2013). Stability analysis of high-order Hopfield-type neural networks based on a new impulsive differential inequality, International Journal of Applied Mathematics and Computer Science 23(1): 201-211, DOI: 10.2478/amcs-2013-0016.

Maccari, A. (2001). The response of a parametrically excited van der Pol oscillator to a time delay state feedback, Nonlinear Dynamics 26(2): 105-119.

Palit, A. and Datta, D.P. (2010). On a finite number of limit cycles in a Lienard system, International Journal of Pure and Applied Mathematics 59: 469-488.

Reddy, R.D., Sen, A. and Johnston, G. (2000). Experimental evidence of time-delay-induced death in coupled limit-cycle oscillators, Physical Review Letters 85(3381): 3381-3384.

Xu, J. and Chung, K. (2003). Effects of time delayed position feedback on a van der Pol-Duffing oscillator, Physica $D$ 180(1): 17-39.

Yu, W. and Cao, J. (2005). Hopf bifurcation and stability of periodic solutions for van der Pol equation with time delay, Nonlinear Analysis 62: 141-165.
Żebrowski, J., Kuklik, P. and Baranowski, R. (2008). Relaxation oscillations in the atrium-a model, Proceedings of the 5th Conference of the European Study Group on Cardiovascular Oscillations, Parma, Italy, pp. 04:16-04:19.

Zhou, X., Jiang, M. and Cai, X. (2011). Hopf bifurcation analysis for the van der Pol equation with discrete and distributed delays, Discrete Dynamics in Nature and Society, 2011: 1-8, Article ID: 569141.

Beata Zduniak received the M.Sc. degree in mathematics from Cardinal Stefan Wyszyński University, Poland, in 2007, the M.Sc. degree in physics from the Warsaw University of Technology, Poland, in 2008, and the Ph.D. degree in mechanics from the Military University of Technology, Poland, in 2011. She is currently an assistant professor of the Warsaw University of Life Sciences, Poland. Her research interests include dynamical systems, delayed differential equations, biomathematics and mechanics.

Marek Bodnar is an assistant professor in the Institute of Applied Mathematics and Mechanics in the University of Warsaw. He received the M.Sc. degree in applied mathematics as well as the Ph.D. and D.Sc. degrees in mathematics from the Faculty of Mathematics, Informatics and Mechanics, University of Warsaw, in 1998, 2001 and 2012, respectively. His research interests include differential equations with delay, dynamical systems and their application to mathematical models describing biological phenomena.

Urszula Foryś is a professor in the Institute of Applied Mathematics and Mechanics, University of Warsaw. She received the M.Sc. degree in applied mathematics and the Ph.D. degree in mathematics from the Faculty of Mathematics, Informatics and Mechanics, University of Warsaw, in 1989, 1996, respectively, while the D.Sc. degree in biocybernetics and biomedical engineering was granted to her by the Polish Academy of Sciences in 2007. Her research interests include dynamical systems, especially differential equations with delay, with applications to mathematical modelling of various natural phenomena, mainly from biology, medicine and sociology.

Received: 2 October 2013 Revised: 8 January 2014 